\title{
Guidelines
}

\section{ASIPP Guidelines for Sedation and Fasting Status of Patients Undergoing Interventional Pain Management Procedures}

\begin{abstract}
Alan D. Kaye, MD, PhD', Mark R. Jones, MD², Omar Viswanath, MD³, Kenneth D. Candido, MD4, Mark V. Boswell, MD, PhD ${ }^{5}$, Amol Soin, MD ${ }^{6}$, Mahendra Sanapati, MD, Michael E. Harned, MD, Thomas T. Simopoulos, MD², Sudhir Diwan, MD ${ }^{9}$, Sheri L. Albers, DO ${ }^{10}$, Sukdeb Datta, MD ${ }^{11}$, Frank J.E. Falco, MD ${ }^{12}$, and Laxmaiah Manchikanti, MD ${ }^{13}$
\end{abstract}

From: ${ }^{1}$ Louisiana State University Health Science Center, New Orleans, LA; ${ }^{2}$ Beth Israel Deaconess Medical Center, Harvard Medical School, Boston, MA; ${ }^{3}$ Valley Anesthesiology and Pain Consultants, University of Arizona College of Medicine, Creighton University School of Medicine, Phoenix, AZ; ${ }^{4}$ Advocate Illinois Masonic Medical Center and University of Illinois College of Medicine, Chicago, IL; 5 University of Toledo College of Medicine and Life Sciences, Toledo, $\mathrm{OH} ;{ }^{6}$ Ohio Pain Clinic, Centerville, $\mathrm{OH}$, Wright State University, Dayton, $\mathrm{OH}$, and Ohio University College of Medicine, Athens, $\mathrm{OH}$; ${ }^{7}$ Pain Management Centers of America, Evansville, IN and Advanced Care

Pain Clinic, Evansville, IN; ${ }^{8}$ University of Kentucky College of Medicine, Lexington,

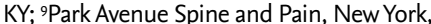

NY; ${ }^{10}$ Radiology Research and Consultation, Sacramento, CA; "Datta Endoscopic Back Surgery and Pain Center and Mount Sinai School of Medicine, New York, NY; ${ }^{12}$ Mid Atlantic Spine \& Pain Physicians, Newark, DE, and Temple University Hospital, Philadelphia, PA; ${ }^{13}$ Pain Management Centers of America, Paducah, KY.

Address Correspondence: Laxmaiah Manchikanti, MD 2831 Lone Oak Road

Paducah, Kentucky 42003 E-mail:drlm@thepainmd.com

Conflict of interest: See P.

Manuscript received: 03-12-2019 Revised manuscript received: 04-01-2019 Accepted for publication: 04-04-2019

Free full manuscript: www.painphysicianjournal.com
Many of the patients undergoing interventional procedures have daily regimens of medications including analgesics, muscle relaxants, and other drugs that can have significant additive/synergistic effects during the perioperative period. Further, many patients also present with comorbid states, including obesity, cardiovascular, and pulmonary disease. Consequently, in the perioperative period, a significant number of patients have suffered permanent neurologic injury, hypoxic brain injury, and even death as a result of over sedation, hypoventilation, and spinal cord injury. In addition, physicians are concerned about aspiration, subsequent complications, and as a result, they ask patients to fast for several hours prior to the procedures.

Based on extensive literature and consensus, a minimum fasting period is established as 2 hours before a procedure for clear liquids and 4 hours before procedure for light meals, rather than having all patients fast for 8 hours or even fasting beginning at midnight the night before the procedure. Gastrointestinal stimulants, gastric acid secretion blockers, and antacids may be used, even though not routinely recommended.

Due to the nature of chronic pain and anxiety, many patients undergoing interventional techniques may require mild to moderate sedation. Deep sedation and/or general anesthesia for most interventional procedures is considered as unsafe, since the patient cannot communicate acute changes in symptoms, thus, resulting in morbidity and mortality, as well as creating compliance issues. We are adapting the published standards of the American Society of Anesthesiologists for monitoring patients under sedation, regardless of the location of the procedure, either office-based, in a surgery center, or a hospital outpatient department. These standards include monitoring of blood pressure, cardiac rhythm, temperature, pulse oximetry, and continuous quantitative end tidal $\mathrm{CO} 2$ monitoring. Sedation must be provided either by qualified anesthesia or non-anesthesia providers, with appropriate understanding of the medications, drug interactions, and resuscitative protocols.

Key words: Guidelines, sedation, fasting status, monitoring, neurological complications

Pain Physician 2019: 22:201-207

\section{BACKGROUND}

Tens of thousands of patients undergo interventional pain procedures annually in the U.S. (1-6). Many of these patients have daily regimens of medications including analgesics, muscle relaxants, and other drugs that can have significant additive/synergistic effects during the perioperative period. Additionally, many of these patients have co-morbid states, including obesity, 
obstructive sleep apnea, cardiovascular and pulmonary disease. Patients have suffered permanent neurologic injury, hypoxic brain injury, and even death as a result of over sedation, hypoventilation, and spinal cord injury (7-14). The American Society of Interventional Pain Physicians (ASIPP) has developed guidelines for the best practice delivery of sedation for interventional pain procedures (15-17).

Three major potential complications associated with the use of sedation and anesthesia for procedures should be appreciated by every interventional pain practitioner. First, if a needle is inserted incorrectly in a location such as the spinal cord, the patient will not be able to communicate sudden paresthesias, dysesthetic pain, or any other aberrant sensations to the interventional pain practitioner while under deep sedation or general anesthesia. This feedback information from the patient can be vital to reduce the potential for severe and permanent complications during and interventional procedure. Needle penetration into the spinal cord itself may not cause pain or irreversible damage; rather, intramedullary deposition of as little as $0.5-1 \mathrm{~mL}$ of injectate will most likely result in neurologic deficit due to syrinx formation and disruption of neuronal pathways $(13,18)$. The second potential complication is related to the requirement of prone positioning for most interventional pain procedures, which may incur additional risk and complicate the provider response when adverse events do occur. Thirdly, the inherent risk of deep sedation and/or general anesthesia per se should be considered, which can result in hypoventilation, pulmonary compromise, anoxic brain states, and/ or death.

In addition to the above complications, physicians should be concerned about aspiration risk and complications related to keeping patients NPO for the required period of time prior to the procedure.

It should be noted that under appropriate circumstances, properly managed sedation can improve patient comfort and facilitate performance of interventional procedures. However, given the inherent hazards, particularly with deep sedation and general anesthesia, the risk versus benefit ratio for specific patients should be assessed. It would not seem prudent to use sedation for every patient without appropriate evaluation or based solely on revenue considerations for the practice.

\section{Fasting Status}

In 1883 Lister (19) recommended that patients may drink clear liquids about 2 hours before surgery, but there should be no solid matter in the stomach. For the next 80 years, textbook fasting guidelines were 2 to 3 hours for clear liquids and 4 to 6 hours for easily digestible foods. In 2017, the American Society of Anesthesiologists (ASA) published an updated report on Practice Guidelines for preoperative fasting and the use of pharmacologic agents to reduce the risk of pulmonary aspiration (20). They defined perioperative pulmonary aspiration as aspiration of gastric contents occurring after induction of anesthesia, during a procedure, or in the immediate postoperative period. They also defined preoperative fasting as a prescribed period before a procedure when patients are forbidden from oral intake of liquids or solids. Their recommendations included limiting clear liquids to 2 hours before and light meals 6 hours before anesthesia, in line with the earlier recommendations of 4 to 6 hours.

A single study available in the interventional pain management literature (21) demonstrated a lack of complications in patients without fasting and undergoing sedation for interventional techniques. The authors concluded that postoperative nausea, vomiting, and respiratory depression are rare and aspiration almost nonexistent, despite almost all patients receiving sedation without preoperative fasting prior to provision of the interventional techniques. In radiology, a prospective observational study (22) showed an extremely low incidence of nausea and vomiting of $0.071 \%$ and no aspiration with enhanced CT examination. They concluded that the occurrence of nausea and vomiting has no correlation with the preoperative solid food consumption status. In contrast, an assessment of patients exposed to non-ionic contrast media (23) examined the incidence and risk factors for nausea and vomiting associated with preparatory fasting. The results showed mild nausea occurring in $2.9 \%$ of patients and no vomiting with a 6 hour preparatory fast from solid food. Many patients underwent excessive fasting for fluids, as well as solid food, and their fasting durations were not associated with the development of peri-procedural nausea. However, they also showed that a history of drug hypersensitivity was an independent risk factor for nausea. In an earlier manuscript, which assessed the relationship between oral food intake and nausea caused by intravenous injection of iodinated contrast material, the incidence of nausea was $6.7 \%$ in the high osmolarity contrast medium group and $1.4 \%$ in the low osmolarity contrast medium group (24). However, the incidence of nausea and vomiting increased with 
the interval between the oral intake of food and the intravenous injection of contrast medium, leading to the conclusion that fasting before contrast computed tomography (CT) enhanced the adverse effects of nausea and vomiting, rather than reducing it.

Based on extensive literature and the ASA recommendations, for patients undergoing sedation, a minimum fasting period of 2 hours for clear liquids and 4 hours for a light meal should be established rather than NPO for 8 hours or requiring that all patients remain NPO after midnight. Gastrointestinal stimulants such as metoclopramide, gastric acid secretion blockers such as cimetidine, famotidine, ranitidine, omeprazole, and lansoprazole may be used but are not routinely recommended (20). Antacids, sodium citrate, sodium bicarbonate, magnesium trisilicate, antiemetics, and ondansetron may be used as monotherapy or in combination but are not recommended as empiric therapies (20).

\section{LeVEls of Sedation for InTERVEntional Pain Procedures}

Numerous publications, though not extensive (2129), have provided discordant information in reference to the need for sedation during interventional pain management techniques; however, multiple LCDs, medical guidelines, and society guidelines describe their policies for sedation for interventional pain procedures with many patients requiring only local anesthesia or mild sedation (29-40). Given that patients undergoing pain procedures typically are anxious and in pain, mild to moderate sedation is acceptable for many patients. Deep sedation and/or general anesthesia for most interventional pain procedures may be unsafe because the patient cannot communicate injury-related pain (e.g, spinal cord injection), which can result in morbidity and/or mortality as well as create compliance issues. Another major concern is that most patients undergoing interventional pain procedures are taking potent medications that possess synergistic sedative-hypnotic properties. Drug-drug interactions may result in over sedation and complications. Deeper sedation should only be undertaken in the presence of anesthesia providers and for patients who have high anxiety, complex pharmacotherapy, or a low pain threshold when undergoing more painful interventional pain procedures. In special cases, the risk of patient movement during a procedure resulting in potential inadvertent injury may justify a deeper anesthetic state.

\section{Monitoring}

The ASA has published standards for monitoring patients under sedation, regardless of location of a procedure, e.g., office based, surgery center, and hospital. These standards are identical regardless of the setting and require blood pressure monitoring, assessment of real-time EKG, temperature measurement (if indicated or anticipated variance), pulse oximetry, and continuous quantitative end tidal $\mathrm{CO} 2$ monitoring. The omission of end tidal $\mathrm{CO} 2$ monitoring can significantly delay identification of hypoventilation and appropriate airway support, and result in catastrophic complications including anoxic brain injury and even death (29,41-43). Supplemental oxygen by nasal cannula is also recommended, particularly for deep sedation (44).

\section{Sedative Medications}

i. Providers: Qualified anesthesia and non-anesthesia providers administer sedation for patients for a variety of diagnostic, therapeutic, and/or surgical interventional pain procedures. Practitioners should aim to provide patients with the benefits of sedation and/or analgesia while minimizing the associated risks. Individuals responsible for patients receiving sedation and/or procedural analgesia should understand the pharmacology of the agents being administered as well as the role of pharmacologic antagonists for opioids and benzodiazepines to rescue patients.

ii. Sedation Goals for Interventional Pain Procedures: Combinations of sedative-hypnotics and analgesics should be administered in a titrated fashion, using as little as is reasonable to accomplish the stated goals, and as appropriate for the procedure being performed and the condition of the patient. Many patients undergoing interventional pain procedures only require local anesthesia infiltration and no additional sedation. Mild to moderate sedation is acceptable in carefully selected patients. Deep sedation and/or general anesthesia, and the drugs most commonly utilized for such purposes (e.g. propofol, ketamine, and etomidate), are not appropriate for most interventional pain procedures (44).

iii. Drug Interactions: Potential drug interactions require the clinician providing sedation to appreciate potential drug-drug effects, which can lead to morbidity and mortality, primarily from cardiorespiratory depression. Additive and/or synergistic 
effects of two or more sedatives are well described and may result in central nervous system and respiratory depression. Anesthesia providers spend years studying and refining their practice; this is not always the case for the non-anesthesia sedation provider. Therefore, it is strongly recommended that healthcare providers develop a comprehensive understanding of the side effects as well as potential drug-drug interactions of the agents they are using in each clinical setting. This must include over the counter agents and herbals, of which there are over 29,000 available, many of which can interact in unfavorable ways with conventional sedative medications (45-48).

\section{VI/ Drug Selection}

i. Benzodiazepines possess anxiolytic, amnesic, and sedative properties (49). Commonly administered benzodiazepines include midazolam (Versed), diazepam (Valium), and lorazepam (Ativan). The most commonly used benzodiazepine for sedation is midazolam. It is rapidly redistributed from the brain to other tissues and metabolized by the liver. Thus, it has a short duration of action. Metabolism in the liver is by hydroxylation, and midazolam is excreted by the kidneys after conjugation. Terminal elimination half-time of midazolam is about 1-4 hours. The elimination half-time may be doubled in the elderly as a result of age-related decreases in hepatic blood flow and possibly by enzymatic activity. Midazolam should be used with caution in the morbidly obese related to their increased volume of distribution and its prolonged half-life.

ii. Opioids are potent analgesics commonly used to provide analgesia before, during, and/or after procedures. Opioids are often utilized in combination with benzodiazepines to provide sedation and analgesia. Some of the more commonly used opioid agonists are morphine, fentanyl (Sublimaze), hydromorphone (Dilaudid) and meperidine (Demerol). In the setting of interventional pain procedures, fentanyl's analgesic effects are 75-125 times more potent on a mg-mg basis than morphine (50). Fentanyl is often used as the analgesic component in sedation because of its rapid onset of clinical action and minimal histamine release. A single dose of fentanyl IV has a more rapid onset and shorter duration of action than an equianalgesic dose of morphine.
The greater lipid solubility of fentanyl compared to morphine explains its more rapid onset and greater potency. The short duration of a single dose of fentanyl associated with a rapid fall in plasma concentration reflects its rapid redistribution into inactive tissue sites such as skeletal muscle and fat. However, when multiple IV doses of fentanyl are administered, or with continuous infusion, progressive saturation of inactive tissues occurs. As a result, duration of analgesia and depression of ventilation may be prolonged when the plasma concentrations of the drug do not decrease rapidly. Metabolism of fentanyl occurs in the liver. IV fentanyl results in clinical effects within 30 seconds to 1 minute. Peak effects occur within 10 minutes and duration of action is 30-60 minutes following a single dose. Analgesic concentrations of fentanyl greatly potentiate the effects of midazolam. Synergism of opioids and benzodiazepines plays an important role in achieving hypnotic states for potentially painful procedures. However, the combination can easily result in respiratory depression (51). Chest wall rigidity has been reported in adults receiving analgesic doses of fentanyl and is best avoided by slow injection of low doses (52).

iii. Propofol (Diprivan) is not recommended for interventional pain procedures because of its potency, which can result in rapid deep sedation and/or general anesthesia states, making the patient unable to communicate when a needle is inadvertently placed incorrectly as well as resulting in dose-dependent respiratory depression. Propofol is a short-acting sedative-hypnotic agent with antiemetic properties (53). Propofol is highly lipid-soluble, which explains the drug's rapid onset. Propofol is well suited for deep sedation because it allows for prompt recovery without residual sedation and a low incidence of nausea and vomiting. Even at low doses, propofol can cause decreased oxygen levels, increased carbon dioxide levels, and inhibit airway reflexes. Because of its pronounced respiratory depressant effects and narrow therapeutic range, propofol should only be administered by individuals trained in airway management and should not be utilized for interventional pain procedures under mild to moderate sedation. In this regard, dosing regimens should be further modified for sedation in elderly patients and those with severe systemic disease. 


\section{Conclusion:}

Sedation for interventional pain procedures should be done with caution and, if deeper sedation is required, the interventional pain physician must take precautions to protect their patients from the risks of over sedation. This includes ensuring that personnel skilled in airway management are available to resolve potential complications, anticipate over-sedation and maintain antagonists and reversal agents readily at hand, and consider neuromonitoring during procedures requiring deep sedation which incur heightened risk of neurologic injury. Appropriate vital sign monitoring should be considered for patients receiving sedation, including blood pressure, EKG, temperature, pulse oximetry, and continuous quantitative end-tidal $\mathrm{CO} 2$ monitoring. The availability of supplemental oxygen should be easily accessible or given prophylactically to patients who are sedated. In preparation for sedation, the interventional physician should assess the NPO status of their patients and have a protocol in place to reduce the risk of aspiration complications. The minimum recommended NPO status is 2 hours for clear liquids and 4 hours for a light meal in lieu of NPO after midnight for all patients.

\section{ACKNOWLedgments}

Dr. Kaye is Professor, Program Director, and Chairman, Department of Anesthesiology, Louisiana State University Health Science Center, New Orleans, LA.

Dr. Jones, Department of Anesthesia, Critical Care and Pain Medicine, Beth Israel Deaconess Medical Center, Harvard Medical School, Boston, MA.

Dr. Viswanath, Valley Anesthesiology and Pain Consultants, Clinical Assistant Professor of Anesthesiology, University of Arizona College of Medicine, Clinical Assistant Professor of Anesthesiology, Creighton University School of Medicine, Phoenix, AZ.

Dr. Candido is Chairman, Department of Anesthesiology, Advocate Illinois Masonic Medical Center, Professor of Clinical Anesthesiology, and Clinical Professor of Surgery, University of Illinois College of Medicine, Chicago, IL.

Dr. Boswell is Professor and Program Director, Pain Medicine, Department of Anesthesiology, University of Toledo College of Medicine and Life Sciences, Toledo, $\mathrm{OH}$.

Dr. Soin is Medical Director, Ohio Pain Clinic, Centerville, $\mathrm{OH}$, Clinical Assistant Professor of Surgery at Wright State University, Dayton, $\mathrm{OH}$, and Assistant Professor,
Ohio University College of Medicine, Athens, $\mathrm{OH}$

Dr. Sanapati is Co-Director, Pain Management Centers of America, Evansville, IN, and Medical Director, Advanced Care Pain Clinic, Evansville, IN

Dr. Harned is Associate Professor with the Department of Anesthesiology, Division of Pain Medicine, and Division Chief of Interventional Pain Medicine at the University of Kentucky College of Medicine, Lexington, KY.

Dr. Simopoulos is Assistant Professor, Department of Anesthesia, Critical Care and Pain Medicine, Beth Israel Deaconess Medical Center, Harvard Medical School, Boston, MA.

Dr. Diwan is President, Park Avenue Spine and Pain, New York, NY

Dr. Albers is Director of Research, Radiology Research and Consultation, Sacramento, CA.

Dr. Datta is Medical Director, Datta Endoscopic Back Surgery and Pain Center and Professorial Lecturer, Department of Anesthesiology, Mount Sinai School of Medicine, New York, NY.

Dr. Falco is Medical Director of Mid Atlantic Spine \& Pain Physicians, Newark, DE; Director, Pain Medicine Fellowship Program, Temple University Hospital, Philadelphia, PA; and Adjunct Associate Professor, Department of PM\&R, Temple University Medical School, Philadelphia, PA.

Dr. Manchikanti is Co-Director, Pain Management Centers of America, Paducah, KY, Medical Director of the Pain Management Center of Paducah, Paducah, KY, Clinical Professor, Anesthesiology and Perioperative Medicine, University of Louisville, Louisville, KY, and Professor of Anesthesiology-Research, Department of Anesthesiology, School of Medicine, LSU Health Sciences Center, New Orleans, LA.

\section{Conflict of Interest}

Dr. Kaye is a speaker for Merck.

Dr. Harned receives payment for development of educational presentations from Medtronic and honorarium from St. Jude Medical.

Dr. Simopoulos is a consultant for Nevro Corp, St. Jude Medical, and Boston Scientific.

Dr. Datta receives research support from Sucampo Pharmaceuticals and an honorarium from Smith and Nephew

Dr. Falco is a consultant for St. Jude Medical Inc. and Joimax Inc. 


\section{References}

1. Manchikanti L, Soin A, Mann DP, Bakshi S, Pampati V, Hirsch JA. Reversal of growth of utilization of interventional techniques in managing chronic pain in medicare population post Affordable Care Act. Pain Physician 2017; 20:551-67.

2. Manchikanti L, Soin A, Mann DP, Bakshi S, Pampati V, Hirsch JA. Comparative analysis of utilization of epidural procedures in managing chronic pain in the Medicare population: Pre and post Affordable Care Act. Spine (Phila Pa 1976) 2019; 44:220-232.

3. Hirsch JA, Chandra RV, Pampati V, Barr JD, Brook AL, Manchikanti L. Analysis of vertebral augmentation practice patterns: A 2016 update. J Neurointer Surg 2016; 8:1299-1304.

4. Manchikanti L, Pampati $V$, Benyamin RM, Hirsch JA. Cost calculation methodology exacerbates site-of-service differentials by $10-$ to 18 -fold for soft tissue and joint injections in hospital outpatient departments. IPM Reports 2017; 1:183-189.

5. Thomson S. Spinal cord stimulation's role in managing chronic disease symptoms. International Neuromodulation Society, November 14, 2016.

https://www.neuromodulation.com/ spinal-cord-stimulation

6. Lad SP, Babu R, Bagley JH, Choi J, Bagley CA, Huh BK, Ugiliweneza B, Patil CG, Boakye M. Utilization of spinal cord stimulation in patients with failed back surgery syndrome. Spine (Phila Pa 1976) 2014; 39:E719-E727.

7. Rathmell JP, Michna E, Fitzgibbon DR, Stephens LS, Posner KL, Domino KB. Injury and Liability Associated with Cervical Procedures for Chronic Pain. Anesthesiology 2011; 114:918-926.

8. Bhananker SM, Posner KL, Cheney FW Caplan RA, Lee LA, Domino KB. Injury and liability associated with monitored anesthesia care: a closed claims analysis. Anesthesiology 2006; 104:228-234.

9. Heran MKS, Smith AD, Legiehn GM. Spinal injection procedures: A review of concepts, controversies, and complications. Radiol Clin North Am 2008; 46:487-514.

10. Bartynski WS, Grahovac SZ, Rothfus WE. Incorrect needle position during lumbar epidural steroid administration: inaccuracy of loss of air pressure resistance and requirement of fluoroscopy and epidurography during needle insertion. AJNR Am J Neuroradiol 2005;
26:502-505.

11. Cucuzzella TR, Delport EG, Kim N, Marley J, Pruitt C, Delport AG. A survey: Conscious sedation with epidural and zygapophyseal injections: is it necessary? Spine J 2006; 6:364-369.

12. Lee J-H, Lee J-K, Seo B-R, Moon S-J, Kim J-H, Kim S-H. Spinal cord injury produced by direct damage during cervical transforaminal epidural injection. Reg Anesth Pain Med 2008; 33:377-379.

13. Epstein N. Neurological complications of lumbar and cervical dural punctures with a focus on epidural injections. Surg Neurol Int 2017; 8:60.

14. Elder BD, Goodwin CR, Abu-Bonsrah N, Sciubba DM. Intramedullary steroid injection of the cervical spine with incidental vertebral artery dissection. Spine J 2016; 16:e725-e726.

15. The National Uniform Claims Committee. Specialty Designation for Interventional Pain Management- oو.

www.cms.hhs.gov/transmittals/Downloads/r1779b3.pdf

16. Medicare Payment Advisory Commission. 2001. Report to the Congress: Paying for interventional pain services in ambulatory settings. Washington, DC: MedPAC. December 2001.

www.medpac.gov

17. Manchikanti L, Abdi S, Atluri S, Benyamin RM, Boswell MV, Buenaventura RM, Bryce DA, Burks PA, Caraway DL, Calodney AK, Cash KA, Christo PJ, Cohen SP, Colson J, Conn A, Cordner HJ, Coubarous S, Datta S, Deer TR, Diwan SA, Falco FJE, Fellows B, Geffert SC, Grider JS, Gupta S, Hameed H, Hameed $M$, Hansen $H$, Helm II S, Janata JW, Justiz R, Kaye AD, Lee M, Manchikanti KN, McManus CD, Onyewu O, Parr AT, Patel V, Racz GB, Sehgal N, Sharma M, Simopoulos TT, Singh V, Smith HS, Snook LT, Swicegood J, Vallejo R, Ward SP, Wargo BW, Zhu J, Hirsch JA. An update of comprehensive evidence-based guidelines for interventional techniques of chronic spinal pain: Part II: Guidance and recommendations. Pain Physician 2013; 16:S49-S283.

18. Bose B. Quadriparesis following cervical epidural steroid injections: case report and review of the literature. Spine ] 2005; 5:558-563.

19. Lister J. On Anaesthetics. In: Holmes system of surgery. 3rd ed. London: Longmans Green and Co; 1883.
20. Practice guidelines for preoperative fasting and the use of pharmacologic agents to reduce the risk of pulmonary aspiration: Application to healthy patients undergoing elective procedures: An updated report by the American Society of Anesthesiologists Task Force on Preoperative Fasting and the use of pharmacologic agents to reduce the risk of pulmonary aspiration. Anesthesiology 2017; 126:376-393.

21. Manchikanti L, Malla Y, Wargo BW, Fellows B. Preoperative fasting before interventional techniques: Is it necessary or evidence-based? Pain Physician 2011; 14:459-467.

22. Li X, Liu H, Zhao L, Liu J, Cai L, Zhang L, Liu L, Zhang W. The effect of preparative solid food status on the occurrence of nausea, vomiting and aspiration symptoms in enhanced CT examination: prospective observational study. $\mathrm{Br}$ J Radiol 2018; 91:20180198.

23. Kim YS, Yoon SH, Choi YH, Park CM, Lee W, Goo JM. Nausea and vomiting after exposure to non-ionic contrast media: incidence and risk factors focusing on preparatory fasting. $\mathrm{Br}$ J Radiol 2018; 91:20180107.

24. Oowaki K, Saigusa H, Ojiri H, Ariizumi M, Yamagisi J, Fukuda K, Tada S. Relationship between oral food intake and nausea caused by intravenous injection of iodinated contrast material. Nihon Igaku Hoshasen Gakkai Zasshi 1994; 54:476-479.

25. Ahmed SU, Tonidandel W, Trella J, Martin NM, Chang Y. Peri-procedural protocols for interventional pain management techniques: a survey of US pain centers. Pain Physician 2005; 8:181-185.

26. Kim N, Delport E, Cucuzzella T, Marley J, Pruitt $C$. Is sedation indicated before spinal injections? Spine (Phila Pa 1976) 2007; 32:E748-E752.

27. Moran TC, Kaye AD, Mai AH, Bok LR. Sedation, analgesia, and local anesthesia: a review for general and interventional radiologists. Radiographics 2013; 33:E47-E6o.

28. Cucuzzella TR, Delport EG, Kim N, MarleyJ, Pruitt C, Delport AG. A survey: conscious sedation with epidural and zygapophyseal injections: Is it necessary? Spine J 2006; 6:364-369.

29. Standards for Basic Anesthetic Monitoring. American Society of Anesthesiologists (ASA), October 28, 2015.

https://www.asahq.org/standards-andguidelines/standards-for-basic-anes- 
thetic-monitoring

30. Noridian Healthcare Solutions, LLC. Local Coverage Determination (LCD). Lumbar Epidural Injections (L34980). Revision Effective Date: 10/1/2017.

31. Novitas Solutions. Local Coverage Determination (LCD): Transforaminal Epidural, Paravertebral Facet and Sacroiliac Joint Injections (L27512). Effective Date 9/1/2014.

32. CGS Administrators, LLC. Local Coverage Determination (LCD). Lumbar Epidural Steroid Injections (ESI), (L34807). Revision Effective Date: 10/1/2017.

33. Wisconsin Physician Services Insurance Corporation. Local Coverage Determination (LCD). Epidural and Transforaminal Epidural Injections (L34622). Effective Date: 10/1/2015.

34. Palmetto GBA. Local Coverage Determination (LCD): Lumbar Epidural Steroid Injections (L34336). Effective Date: 10/1/2015.

35. First Coast Service Options, Inc. Epidural (L33906). Revision Effective Date: 10/01/2017.

36. Noridian Healthcare Solutions, LLC. Local Coverage Determination (LCD). Facet Joint Injections, Medial Branch Blocks, and Facet Joint Radiofrequency Neurotomy (L34995). Revision Effective Date: 11/02/2016.

37. CGS Administrators, LLC. Local Coverage Determination (LCD). Facet Joint Injections, Medial Branch Blocks, and Facet Joint Radiofrequency Neurotomy (L34832). Effective Date: 10/01/2016.

38. Wisconsin Physician Services Insurance
Corporation. Local Coverage Determination (LCD). Facet Joint Injections, Medial Branch Blocks, and Facet Joint Radiofrequency Neurotomy (L35996). Effective Date: 10/1/2015.

39. Palmetto GBA. Local Coverage Determination (LCD): Paravertebral Facet Joint Block (L33439). Effective Date 10/1/2014.

40. First Coast Service Options, Inc. Paravertebral Facet Joint Blocks (L33930). Revision Effective Date: 03/01/2018.

41. Weaver J. The latest ASA mandate: $\mathrm{CO}(2)$ monitoring for moderate and deep sedation. Anesth Prog 2011; 58:111-112.

42. ASA Upgrades Procedural Sedation Standards: Requirement for Capnography.

www.physio-control.com/WorkArea/DownloadAsset.aspx?id=2147486279

43. Kodali BS. Capnography outside the operating rooms. Anesthesiology 2013; 118:192-201.

44. Practice Guidelines for Moderate Procedural Sedation and Analgesia 2018: A Report by the American Society of Anesthesiologists Task Force on Moderate Procedural Sedation and Analgesia, the American Association of Oral and Maxillofacial Surgeons, American College of Radiology, American Dental Association, American Society of Dentist Anesthesiologists, and Society of Interventional Radiology. Anesthesiology 2018; 128:437-479.

45. Short TG, Chui PT. Propofol and midazolam act synergistically in combination. Br J Anaesth 1991; 67:539-545.

46. Jones MR, Calixto F, Kaye AD. Do herbal supplements create unnecessary risk for patients? You're Wrong, I'm Right: Dueling Authors Reexamine Classic Teachings in Anesthesia. 2016.

47. Abe A, Kaye AD, Gritsenko K, Urman $\mathrm{RD}$, Kaye AM. Perioperative analgesia and the effects of dietary supplements. Best Pract Res Clin Anaesthesiol 2014; 28:183-189.

48. Renilla González A, Lozano MartinezLuengas I; Eva M Benito Martín, Secades González S, Alvarez Pichel I, Alvarez Martinez P, Santamarta Liébana E, Díaz Molina B. Cardiogenic shock following administration of propofol and fentanyl in a healthy woman: A case report. J Med Case Rep 2011; 5:382.

49. Griffin CE, Kaye AM, Bueno FR, Kaye $A D$, Kaye AD. Benzodiazepine pharmacology and central nervous system-mediated effects. Ochsner ] 2013; 13:214-223.

50. Owusu Obeng A, Hamadeh I, Smith M. Review of opioid pharmacogenetics and considerations for pain management. Pharmacother J Hum Pharmacol Drug Ther 2017; 37:1105-1121.

51. Hirschtritt ME, Delucchi KL, Olfson M. Outpatient, combined use of opioid and benzodiazepine medications in the United States, 1993-2014. Prev Med Rep 2018; 9:49-54.

52. Çoruh B, Tonelli MR, Park DR. FentanylInduced Chest Wall Rigidity. Chest 2013; 143:1145-116.

53. Sahinovic MM, Struys MMRF, Absalom AR. Clinical pharmacokinetics and pharmacodynamics of propofol. Clin Pharmacokinet 2018; 57:1539-1558. 
